\title{
Quantification of Candesartan in Mouse Plasma by MALDI-TOFMS and in Tissue Sections by MALDI-Imaging Using the Stable- Isotope Dilution Technique
}

\author{
Toyofumi Nakanishi, ${ }^{* 1}$ Shinji Takai, ${ }^{2}$ Denan Jin, ${ }^{2}$ and Takayuki Takubo ${ }^{1}$ \\ ${ }^{1}$ Department of Clinical and Laboratory Medicine, Osaka Medical College, 2-7 Daigaku-cho, Takatsuki, Osaka 569-8686, Japan \\ ${ }^{2}$ Department of Pharmacology, Osaka Medical College, 2-7 Daigaku-cho, Takatsuki, Osaka 569-8686, Japan
}

\begin{abstract}
To determine the contents of candesartan in mouse plasma, and blood vessel and kidney sliced sections and also better understand its pharmacokinetics, we applied matrix-assisted laser desorption/ionization time-of-flight mass spectrometry (MALDI-TOFMS) and MALDI-imaging mass spectrometry (IMS) with the selected reaction monitoring (SRM) mode using a labeled-internal standard. The results of fundamental examinations showed that the slope of the resulting curves of candesar$\tan$ in the plasma from the equation was 0.91 and the y-intercept was 0.02 . Both intra- and inter-day accuracies $(n=10)$ and the precision of candesartan in the plasma by MALDI-TOFMS with the SRM mode were in the range of 3.4 to $17.3 \%$ and $93.2 \%$, respectively. The detection limit of candesartan in spiked plasma was $0.2 \mathrm{nmol} / \mathrm{L}$. IMS analysis enabled us to clarify distinct spacial time-distribution images in sliced mouse blood vessel and kidney sections although it still needed to improve a protocol of quantification. Typical pharmacokinetic patterns of candesartan were obtained in the plasma and sliced kidney sections, but those in the blood vessel sections gradually increased $24 \mathrm{~h}$ after administration. MALDI-TOFMS and IMS with the SRM mode are powerful tools to identify the spacial distribution and traceability of candesartan in sliced blood vessel and tissue sections as well as in the plasma.
\end{abstract}

Keywords: candesartan, stable-isotope dilution technique, MALDI-imaging, spacial localization, pharmacokinetics

(Received February 21, 2013; Accepted April 22, 2013)

\section{INTRODUCTION}

Candesartan (CAD), an angiotensin receptor blocker (ARB), has the most significant clinical usage in the treatment of hypertension of all grades. ${ }^{1)} \mathrm{CAD}$ is an angiotensin II (AT-II) receptor antagonist with selectivity for the AT-I receptor. ${ }^{2)}$ Despite the availability of the ARBs, morbidity and mortality in patients with congestive heart failure and evolution towards end-stage renal failure chronic nephropathies remains high. ${ }^{3-5)}$ It may be necessary to frequently measure the concentrations of CAD in biological samples because the therapeutic concentration of CAD ranges from a few to hundreds of nanograms per milliliter of plasma. Therapeutic drug monitoring and investigational clinical studies have to benefit from fast, precise, accurate, and analytical methods to reveal significant pharmacokinetic effects.

Several analytical methods have been established for the analysis of the ARBs in various body fluids and organs. ARBs extracted in biological samples have been assayed by liquid chromatography with ultra-violet absorption, fluorescence detection, or mass spectrometry with electrospray ionization (ESI-MS). Moreover, tandem mass spectrometry (MS/MS) with ESI coupled to separation techniques presents obvious advantages including short time development, sensitivity, selectivity, and high throughput. ${ }^{6,7)}$ Although these conventional methods can identify the pharmacokinetic profiles of ARBs in fluids and tissue extracts, there is currently no information on distinct spacial time-distribution images in organs. Caprioli et al ${ }^{8,9)}$ developed powerful tools, imaging mass spectrometry (IMS) by matrix-assisted laser desorption/ionization (MALDI) coupled with time-offlight (TOF) and Fourier transform ion cyclotron resonance (FTICR) mass spectrometers, to directly clarify the spacial distributions as well as quantifications of drugs and their metabolites, lipids, peptides, and proteins in tissue sections.

At the low mass/charge region (less than $\mathrm{m} / \mathrm{z} 1,000$ ) of MALDI-TOFMS analysis as well as this present study, the MALDI mass spectrum always contains a large population of ions from sample matrices and matrix-related adduct clusters and fragments. These co-existing ions with high intensities often interfere with the detection of a target compound in the analytes. Therefore, distinguishing targets ions from these chemical backgrounds is of importance. IMS coupled with MS/MS or selective reaction monitoring (SRM) mode analyses may be free of the unique analytical disadvantages of the MALDI technique as similar to the MALDI-FTICR. IMS also permits the analysis of drugs and their metabolites directly from only one piece of a sliced tissue section without target specific labels or reagents. Recently, some groups demonstrated that the quantitative analysis by MALDI imaging MS/MS using a stable isotopic internal standard enabled us to determine contents of small

* Correspondence to: Toyofumi Nakanishi, Osaka Medical College, 2-7 Daigaku-cho, Takatsuki, Osaka 569-8686, Japan, e-mail: nakanisi@poh.osaka-med.ac.jp Please cite this article as: T. Nakanishi, et al., Quantification of Candesartan in Mouse Plasma by MALDI-TOFMS and in Tissue Sections by MALDI-Imaging Using the Stable-Isotope Dilution Technique, Mass Spectrometry, 2: A0021 (2013); DOI: 10.5702/massspectrometry.A0021 


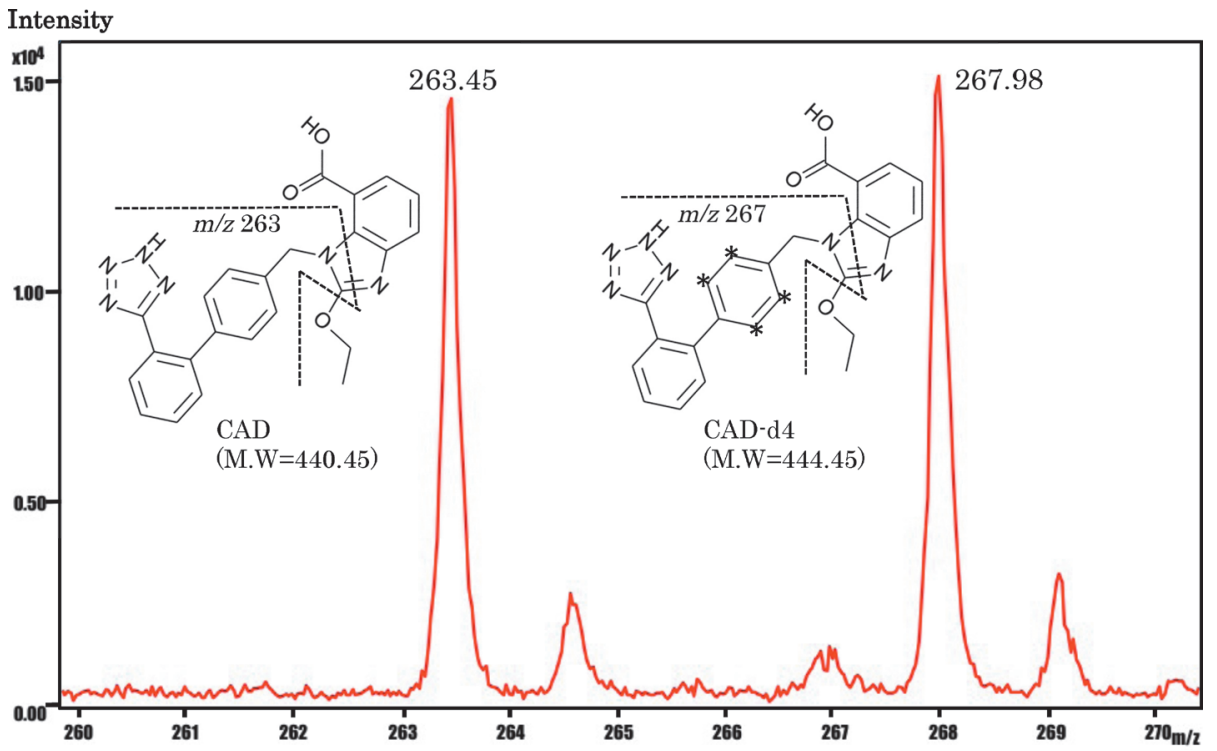

Fig. 1. MALDI post-source decay mass spectrum of CAD and CAD- $d_{4}\left(\mathrm{CAD}^{*}\right)$ in the SRM mode. The monitor ions of CAD and CAD* were $m / z 263.5$ and $m / z 267.5$, respectively.

molecules in animal tissue sections. ${ }^{10-12)}$ By the incorporation of a uniformly applied internal standard coupled with the specificity of MS/MS technique and scan-by-scan ionsignal normalization, the quantitative MALDI imaging was improved in a tissue section.

In the present study, we applied IMS coupled with the SRM mode using a stable isotopic CAD standard to not only determine the contents of CAD in mouse plasma, and blood vessel and kidney sliced sections, but also to better understand its pharmacokinetics. MALDI-TOFMS with the SRM mode gave high intra- and inter-day accuracies and precisions of CAD in the plasma. Using IMS analysis, typical pharmacokinetic images of CAD were obtained in the plasma and sliced kidney section, but those in the sliced blood vessel section gradually increased $24 \mathrm{~h}$ after administration.

\section{EXPERIMENTAL}

\section{Materials}

CAD (purity $=99 \%$ ) was purchased from Takeda Pharmaceutical Co., Ltd. (Osaka, Japan). CAD- $d_{4}$ (purity $>95 \%$ ) as a stable isotope-labeled internal standard was from Clearsynth Labs Pvt. Ltd. (Mumbai, India). Methanol, trifluoroacetic acid (TFA), and acetonitrile were purchased from Nakalai Tesque (Kyoto, Japan). $\alpha$-Cyano-4-hydroxycinnamic acid ( $\alpha$-CHCA) was from Sigma-Aldrich Japan (Tokyo, Japan). An Indium-Tin-Oxide (ITO) coated glass slide (Bruker Daltonics Inc., Billerica, MA, U.S.A.) was used for IMS.

Twenty male mice were purchased from Japan SLC (Shizuoka, Japan). Mice (12-16 wks old) were decapitated, and the blood vessels and kidneys were quickly removed and frozen in liquid nitrogen. Tissues were stored at -80 degrees until they were used for testing. Plasma samples were collected 0-, 1-, 6-, and $24 \mathrm{~h}$ after the oral administration of CAD $(30 \mathrm{mg} / \mathrm{kg})$. All experimental protocols were approved by the Osaka Medical College Animal Care and Use Committee.

\section{Sample preparations}

For MALDI-TOFMS analysis, an aliquot of mouse plasma 200 times diluted with distilled water was added to an equivalent volume of methanol.

Five microliters of the MALDI matrix consisting of $7 \mathrm{mg} /$ $\mathrm{mL}$ of $\alpha$-CHCA in 60\% HPLC-grade acetonitrile and $0.2 \%$ TFA was directly mixed with $5 \mu \mathrm{L}$ of the analytes, and $2 \mu \mathrm{L}$ of the mixture was then dropped onto the MALDI plate (Bruker Daltonics, Billerica, MA, U.S.A.) and dried under room temperature.

To calculate the absolute recovery, two replicates of spiked plasma samples at different concentration ranges (low and high) of CAD were used. These samples were compared with the methanolic solutions of CAD at the concentrations investigated, which had been processed following the same sample preparation procedure as the plasma samples. MALDI-TOFMS with SRM mode precision was determined on day 1 and on 3 different days. A calibration was performed on each day. Precision was measured using two different plasma samples for each concentration range and each day. Intra- and inter-day accuracies were determined by measuring five replicates for each concentration of CAD.

Mouse blood vessels and kidneys were embedded with a small amount of the TissueTek ${ }^{\mathrm{TM}}$ (Sakura Finetek Japan Co., Ltd., Osaka, Japan) for IMS analysis. Tissue sections, $12 \mu \mathrm{m}$ in thickness, were cut with a Cryostat CM-3050 (Leica Biosystems Inc., Tokyo, Japan) at -20 degrees and were mounted on ITO coated glass slides. After washing with warm distilled water for $30 \mathrm{~s}$, the glass plate was desiccated under a vacuum for $1 \mathrm{~h}$. An $\alpha$-CHCA matrix was coated onto the mouse vessel and kidney sliced sections using Image Prep ${ }^{\mathrm{TM}}$ (Bruker Daltonics) according to the manufacturer's instructions. Spray-coated tissue sections were desiccated at room temperature for 5-10 min prior to MALDI analysis.

For the quantification of CAD with MALDI-TOFMS and IMS, stable isotopic CAD (Candesartan- $d_{4}$ : CAD $^{*}$ ) as an internal standard, $100 \mathrm{nmol} / \mathrm{L}$ of the final concentration, was added to the $\alpha$-CHCA matrix. 


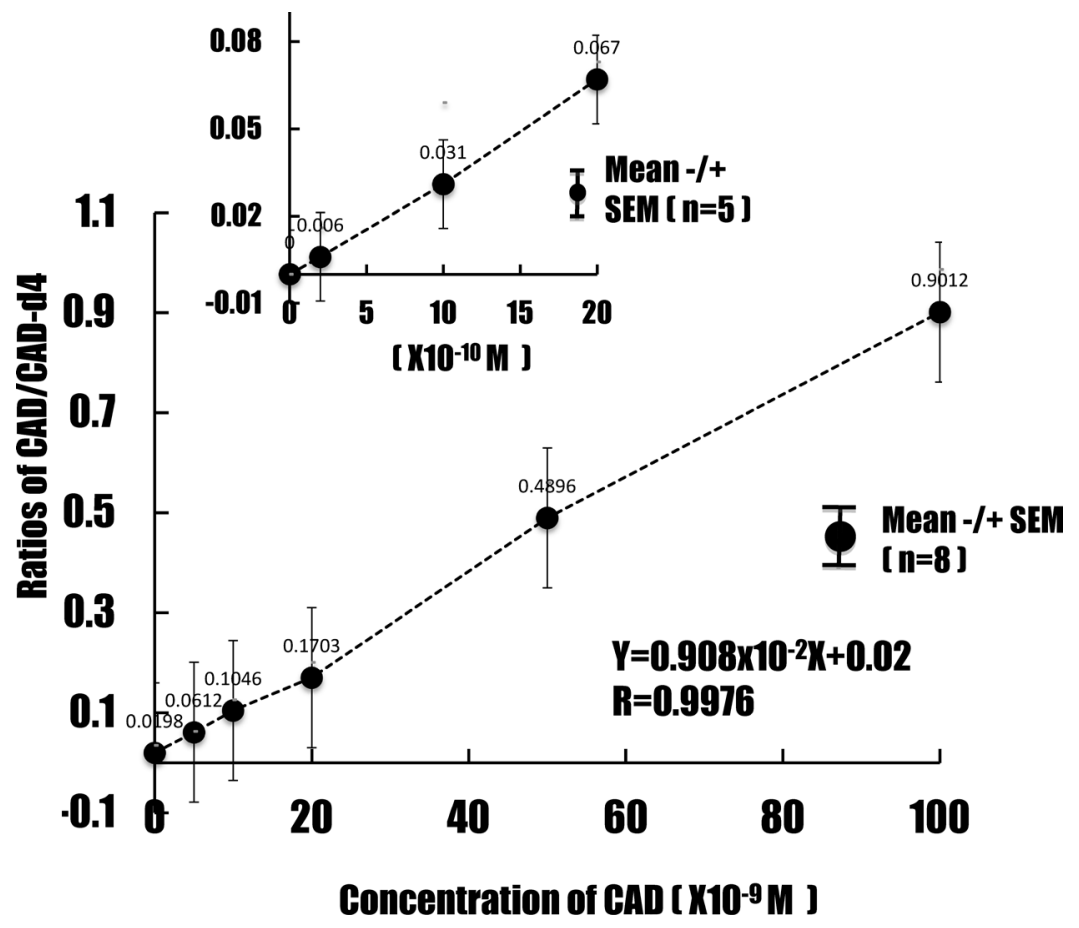

Fig. 2. Calibration curve of CAD contents between $5 \mathrm{nmol} / \mathrm{L}$ to $100 \mathrm{nmol} / \mathrm{L}$. The calibration curve between $0.2 \mathrm{nmol} / \mathrm{L}$ to $2 \mathrm{nmol} / \mathrm{L}$ for the lower range is shown in the inset.

Table 1. Intra-precisions and accuracies of CAD in the plasma by stable-isotope dilution MALDI-TOFMS analysis.

\begin{tabular}{cccc}
\hline & High & Medium & Low \\
\hline 1 & $49.6^{\#}$ & $8.6^{\#}$ & $1.5^{\#}$ \\
2 & 47.7 & 8.2 & 2.1 \\
3 & 52.8 & 6.9 & 1.4 \\
4 & 48.2 & 7.4 & 2.2 \\
5 & 48.9 & 8.3 & 2.0 \\
6 & 49.5 & 7.1 & 1.6 \\
7 & 48.3 & 6.4 & 1.3 \\
8 & 50.2 & 7.7 & 1.9 \\
9 & 51.8 & 8.5 & 1.8 \\
10 & 51.4 & 7.9 & 1.9 \\
\hline \#nmol/mL & & & $1.8^{\#}$ \\
\hline Mean & $49.8^{\#}$ & $7.7^{\#}$ & \\
S.D. & 1.70 & 0.74 & \\
CV (\%) & 3.40 & 9.56 & \\
\hline & & & \\
\hline & & & \\
& & & \\
\hline
\end{tabular}

$\# \mathrm{nmol} / \mathrm{mL}$

\section{MALDI-TOFMS and IMS analyses}

MALDI-TOFMS and IMS analyses were performed on the TOF mass spectrometer Autoflex speed (Bruker Daltonics), which utilized a Smartbeam ${ }^{\mathrm{TM}}$-II solid-state laser (wavelength, $355 \mathrm{~nm}$; focused diameter, $20 \mu \mathrm{m}$; repetition rate, $500 \mathrm{~Hz}$ ).

SRM datasets were acquired under the following conditions: precursor mass, $441.5 \mathrm{Da}$; fragment mass, $263.5 \mathrm{Da}$; ion source, $19.68 \mathrm{kV}$, and reflector voltage, $22.88 \mathrm{kV}$. Other parameters were set as follows: mass range of the parent and fragment ions, $\pm 3.0 \%$; ion selector, $\pm 2.5 \%$ of the parent ion; and window range for the precursor ion, $\pm 7 \mathrm{Da}$.

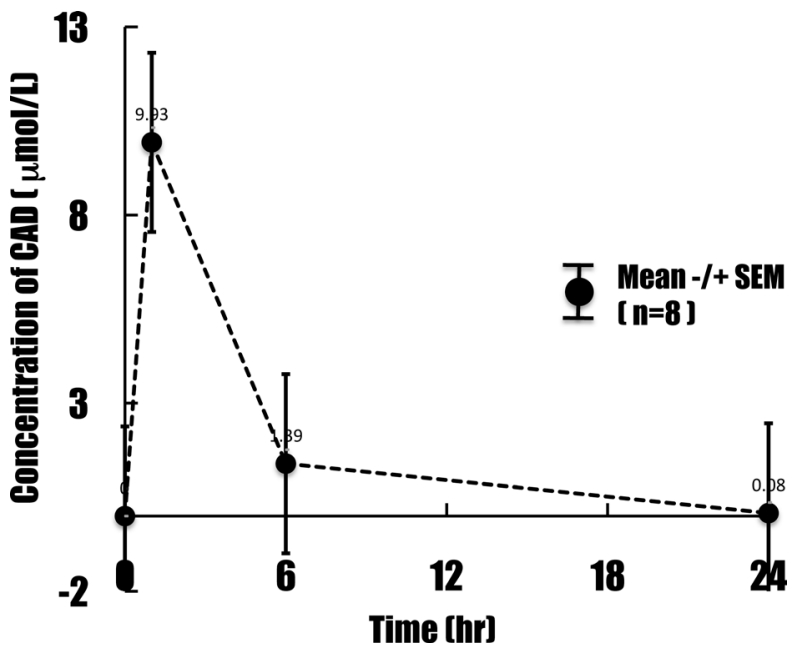

Fig. 3. Typical pharmacokinetic patterns in mouse plasma obtained after a single oral administration $(30 \mathrm{mg} / \mathrm{kg})$.

Spectra were mass calibrated by collecting 500 laser shots of spots containing Bruker peptide calibration standard II, consisting of nine peptide standards and an $\alpha$-CHCA matrix covering a mass range from 190 to $3,200 \mathrm{Da}$.

For MALDI imaging, 500 laser shots were accumulated per pixel, with a raster width of $50 \mu \mathrm{m}$ (laser spot size, $50 \mu \mathrm{m})$. The spacial resolution of ion images was set at $50 \mu \mathrm{m}$; its area is equivalent to $1.96 \times 10^{-3} \mathrm{~mm}^{2}$. Imaging experiments were set up and evaluated using FlexImaging 3.3 software.

\section{RESULTS AND DISCUSSION}

Figure 1 shows a MALDI post-source decay spectrum of CAD itself and stable isotopic CAD by MALDI-TOFMS with the SRM mode. The observed mass at $m / z 263.45$ was 
(a) Scanning images

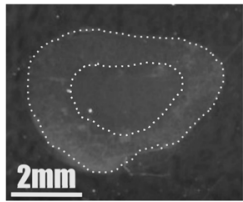

Blank

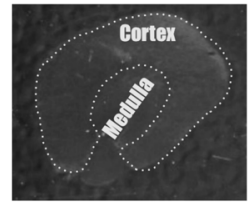

1

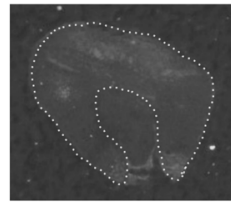

6

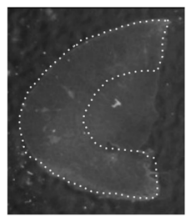

24

Time after the oral administration [hr]

\section{Heat-map images at $m / 2263.5$}

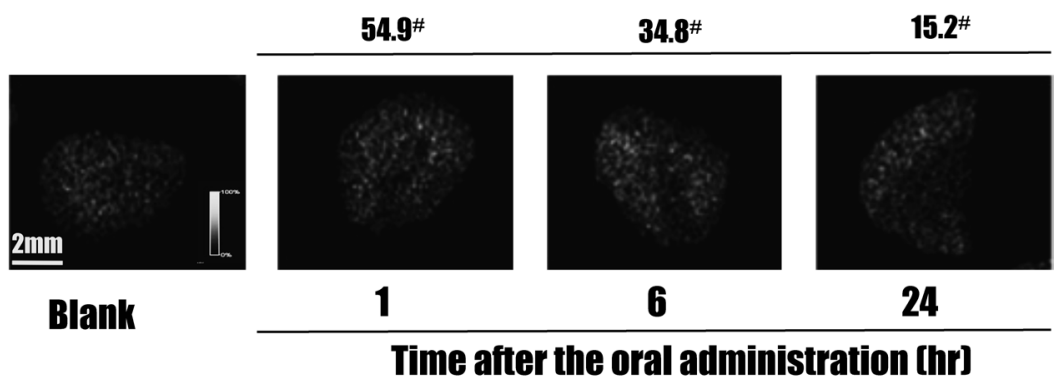

(b) Scanning images

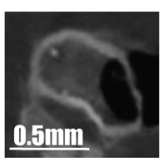

Blank

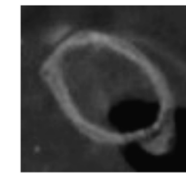

1

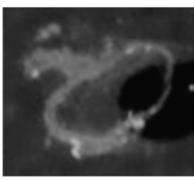

6

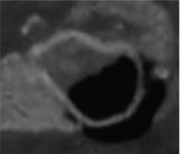

24

\section{Time after oral administration [hr]}

\section{Heat-map images at $m / 2263.5$}

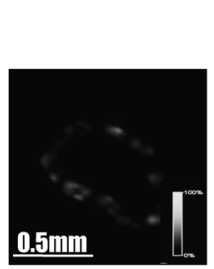

Blank

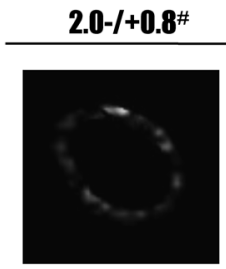

1

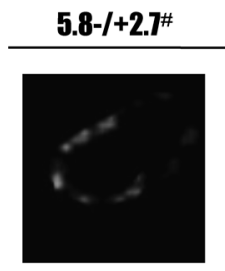

6

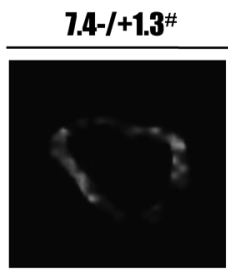

24

Time after oral administration [hr]

Fig. 4. Spacial time-distribution images in sliced mouse kidney (a) and blood vessel (b) sections by IMS with the SRM mode.

Each heat-map of the ion images at $\mathrm{m} / z 263.5$ indicated the contents of CAD in sliced sections $0,1,6$, and $24 \mathrm{~h}$ after the oral administration. Scanning images of each sliced section (upper) and heat-maps of ion images of CAD (lower) are shown. The data for sliced kidney was measured in single and those for blood vessel were in quadruplicate (mean \pm 1 S.D.). \#: $\mathrm{pmol} / \mathrm{mm}^{2}$

a specific fragment ion of CAD itself and $m / z 267.98$ was for stable isotopic CAD. The ratios of intensities between these two monitoring ions were $0.977 \pm 0.009$ (mean \pm S.D., $n=8$ ) at equivalent molar ratios of the mixture.

As shown in Fig. 2, the slope of the resulting curves of CAD in spiked plasma from the equation $(r=0.9976)$ was 0.91 and the $y$-intercept was 0.02 . For intra- $(n=10)$ and inter- $(n=5)$ day precisions, the variability of the results was below the regulatory limits from $3.4 \%$ to $17.2 \%$ for MALDITOFMS with the SRM mode, as shown in Table 1. Accura- cies ranged between $89.0 \%$ and $97.3 \%$, allowing the accurate assay of CAD, as shown in Table 1. A lower range of CAD, below $2.0 \mathrm{nmol} / \mathrm{L}$, could be also calibrated as illustrated in the inset. The quantification limit of CAD in spiked mouse plasma by MALDI-TOFMS with the SRM mode was $0.2 \mathrm{nmol} / \mathrm{L}$. It was approximately 100 times better than that of the other analytical methods. When a comparison was done between the three plasma preparations, filtration using an Ultrafree ${ }^{\mathrm{TM}}$ membrane $(<5 \mathrm{kDa}$, Millipore Co., Ltd., Billerica, MA, U.S.A.), deproteinization with trichloroacetic 
acid, and our protocol, our one-step dilution protocol was confirmed as being at least 10 times higher than that of the others (data not shown). ${ }^{13)}$ Generally, filtration and deproteinization may affect the determination of the contents of medicines and drugs in body fluids because most of them are deposited with plasma proteins, such as albumin.

The concentration-time curve of CAD in mouse plasma $(n=8)$ after a single oral administration of $30 \mathrm{mg} / \mathrm{kg}$ was performed by stable-isotope dilution MALDI-TOFMS with the SRM mode in Fig. 3. After oral administration, the concentration in the plasma rapidly increased, reached a maximum level, $9.33 \pm 0.35 \mu \mathrm{mol} / \mathrm{L}$ (mean \pm S.D.), $1 \mathrm{~h}$ after the oral administration and almost returned to the levels at $0 \mathrm{~h}$ by $24 \mathrm{~h}, 0.08 \pm 0.11 \mu \mathrm{mol} / \mathrm{L}$. Although we did not trace the contents of CAD in the plasma from 1 to $6 \mathrm{~h}$ after the oral administration, the apparent maximum concentration time ( $\left.T_{\max }\right)$ of CAD should be the same as that of the references. ${ }^{14)}$

As shown in Fig. 4, pharmacokinetic distribution images of CAD were obtained in the sliced kidney (a) and blood vessel (b) sections. The spacial distribution image of CAD in the sliced kidney section was relatively concentrated in the cortex region and time-distribution was maximized until $6 \mathrm{~h}$ after the oral administration. The time-distribution pattern was the same as that in the plasma and an apparent $T_{\max }$ was $54.9 \mathrm{pmol} / \mathrm{mm}^{2} 1 \mathrm{~h}$ after the oral administration. On the other hand, that of sliced blood vessel sections increased gradually and very slightly, only to $7.4 \mathrm{pmol} / \mathrm{mm}^{2}$ at the maximum point, until $24 \mathrm{~h}$ after the oral administration because the absorption of CAD into the blood vessels was very low. With the oral administration of CAD in this study, the false-positive signal was still observed with/without a contribution of the content of CAD in a blank tissue section. Pirman et al. reported some studies in which the contents of small molecules in brain tissue sections were determined by quantitative MALDI-MS/MS imaging using the same stable isotope dilution technique and discussed about the origin of a false-positive signal. ${ }^{10,11)}$ It concluded that the similar false-positive signal in the tissue section was the non-labeled target drug with no significant contribution from impurity of the stable isotopic standard. Similarly in our case, the false-positive blank signal in the mouse blood vessel and kidney sections was partially derived from an impurity of deuterated CAD in the same manner, although it was not the same as MALDI matrix in their study. In order to more improve the quantification by this IMS analysis, further study will be needed to identify unknown factor caused the false-positive signal in the tissue sections.

From the results of this pharmacokinetics, it suggests that the transferred CAD into the blood vessel will be still bound with the AT-I receptor in the endothelial cell even though CAD would disappear from the plasma. Although the contents of CAD in these sliced sections were similar to those of tissues extracts using the previous analytical methods, the results obtained still provide valuable information to better understand the pharmacokinetics of CAD. IMS will be enabled to identify more specific localizations of CAD in tissue section, such as a glomerulus, basal lamina and endothelial cell if the spacial resolution would be more improved below $10 \mu \mathrm{m}$.

The present study can be summarized as follows; one-step dilution with methanolic solution, in which filtration or deproteinization to remove proteins was not needed, was an adequate sample preparation for MALDI-TOFMS analysis. Second, extraction and purification of the target from plasma samples could be omitted. Finally, IMS image directly clarified the time-distribution and spacial localization of CAD in the tissue section tested.

In conclusion, MALDI-TOFMS and IMS with the SRM mode coupled with one-step sample preparation were powerful tools to rapidly identify the spacial distribution and traceability of CAD in sliced blood vessel and kidney sections as well as in the plasma, even though a further research will need to clarify an unknown factor caused a falsepositive signal.

\section{Acknowledgements}

We thank Mrs. Minako Ito for providing technical assistance with the tissue sections. This work was partially supported by a Grant-in-Aid for a Joint Project of the Osaka Medical College Center Research Laboratory.

\section{REFERENCES}

1) A. Joost, H. C. Schunkert, P. W. Radke. Candesartan cilexetil: An update. Expert Opin. Pharmacother. 12: 1769-1780, 2011.

2) K. J. McClellan, K. L. Goa. Candesartan cilexetil: A review of its use in essential hypertension. Drugs 56: 847-869, 1998.

3) I. Meineke, H. Feltkamp, A. Högemann, U. Gundert-Remy. Pharmacokinetics and pharmacodynamics of candesartan after administration of its pro-drug candesartan cilexetil in patients with mild to moderate essential hypertension: A population analysis. Eur. J. Clin. Pharmacol. 53: 221-228, 1997.

4) A. Himmelmann, S. Keinänen-Kiukaanniemi, A. Wester, J. Redón, R. Asmar, T. Hedner; Effect Study Group. The effect duration of candesartan cilexetil once daily, in comparison with enalapril once daily, in patients with mild to moderate hypertension. Blood Press. 10: 43-51, 2001.

5) H. Buter, G. Y. Navis, A. J. Woittiez, D. de Zeeuw, P. E. de Jong. Pharmacokinetics and pharmacodynamics of candesartan cilexetil in patients with normal to severely impaired renal function. Eur. J. Clin. Pharmacol. 54: 953-958, 1999.

6) N. Ferreirós, S. Dresen, R. M. Alonso, W. Weinmann. Validated quantitation of angiotensin II receptor antagonists (ARA-II) in human plasma by liquid-chromatography-tandem mass spectrometry using minimum sample clean-up and investigation of ion suppression. Ther. Drug Monit. 29: 824-834, 2007.

7) S. Mehta, R. P. Shah, R. Priyadarshi, S. Singh. LC and LC-MS/ TOF studies on stress degradation behaviour of candesartan cilexetil. J. Pharm. Biomed. Anal. 52: 345-354, 2010.

8) E. H. Seeley, S. R. Oppenheimer, D. Mi, P. Chaurand, R. M. Caprioli. Enhancement of protein sensitivity for MALDI imaging mass spectrometry after chemical treatment of tissue sections. $J$ Am. Soc. Mass Spectrom. 19: 1069-1077, 2008.

9) D. S. Cornett, S. L. Frappier, R. M. Caprioli. MALDI-FTICR imaging mass spectrometry of drugs and metabolites in tissue. Anal. Chem. 80: 5648-5653, 2008.

10) D. A. Pirman, R. F. Reich, A. Kiss, R. M. Heeren, R. A. Yost. Quantitative MALDI tandem mass spectrometric imaging of cocaine from brain tissue with a deuterated internal standard. Anal. Chem. 85: 1081-1089, 2013.

11) D. A. Pirman, R. A. Yost. Quantitative tandem mass spectrometric imaging of endogenous acetyl-L-carnitine from piglet brain tissue using an internal standard. Anal. Chem. 83: 8575-8581, 
2011.

12) L. Signor, E. Varesio, R. F. Staack, V. Starke, W. F. Richter, G. Hopfgartner. Analysis of erlotinib and its metabolites in rat tissue sections by MALDI quadrupole time-of-flight mass spectrometry. J. Mass Spectrom. 42: 900-909, 2007.

13) M. Levi, G. Wuerzner, E. Ezan, A. Pruvost. Direct analysis of valsartan or candesartan in human plasma and urines by on-line solid phase extraction coupled to electrospray tandem mass spectrometry. J. Chromatogr. B Analyt. Technol. Biomed. Life Sci. 877: 919-926, 2009.

14) T. Kondo, K. Ogiwara, Y. Kato, K. Yoshida, Y. Yoshimura, M. Motohashi, K. Tanayama. Jpn. Pharmacol. Therapeutics 24 (Suppl. 6): S915, 1996. 